\title{
DEVELOPMENT OF LIQUISOLID FORMULATION FOR IMPROVED SUSTAINED RELEASE OF PROPRANOLOL HYDROCHLORIDE
}

\author{
ALIYAH ALIYAH*, EMILIA UTOMO, ANDI DIAN PERMANA, ERNAWATI
}

Faculty of Pharmacy, Universitas Hasanuddin, Makassar, Indonesia, 90245

*Email: aliyahputranto@yahoo.co.id

Received: 23 Nov 2020, Revised and Accepted: 21 Jan 2021

\begin{abstract}
Objective: The aim of this study was to develop a liquisolid formulation of propranolol hydrochloride to obtain an improved sustained release profile by varying the ratio of liquid vehicles.

Methods: In this study, propranolol hydrochloride (PPH) was dispersed in the combination of propylene glycol and polysorbate 80, as the liquid vehicles, with different ratios. Eudragit ${ }^{\circledR}$ RL and Aerosil ${ }^{\circledR}$ were used as carrier and coating materials, respectively, to produce a dry and free-flowing powder. In addition, HPMC was used to amplify the retardation effect. The prepared formulations were evaluated for its physicochemical properties, including loss on drying, flow rate, angle of repose calculation, drug content analysis, FT-IR spectroscopy, as well as dissolution studies. The obtained dissolution profiles were subsequently fitted to the mathematical model in order to determine the drug kinetics.

Results: The results show that all formulations performed dry and free-flowing granules containing PPH in the range of 7-9\%. Furthermore, all the prepared formulations were able to sustain the drug release for a total of $8 \mathrm{~h}$ in two different dissolution media, namely simulated gastric fluid and simulated intestinal fluid. F4 containing propylene glycol and polysorbate 80 (1:2) possessed the lowest drug release rate. It was also obtained that F1 and F3 followed first-order kinetics while F2, F4, and F5 complied with the Higuchi model.
\end{abstract}

Conclusion: Overall, there was no difference in all the dissolution profiles based on the calculation of the difference and similarities factor.

Keywords: Liquisolid granules, Propranolol hydrochloride, Sustained release

(C) 2021 The Authors. Published by Innovare Academic Sciences Pvt Ltd. This is an open access article under the CC BY license (https://creativecommons.org/licenses/by/4.0/) DOI: https://dx.doi.org/10.22159/ijap.2021v13i2.40354. Journal homepage: https://innovareacademics.in/journals/index.php/ijap

\section{INTRODUCTION}

During the last decades, conventional dosage forms are rapidly replaced by novel drug delivery systems. Sustained release dosage forms have become one of the most favourable in modern therapeutics [1]. Sustained release drug delivery system is an alternative to maintain therapeutic dose in the systemic after a single application. This can be a solution to optimize the efficacy and safety of the treatment, also reduce the frequency of drug consumption resulting in improving patient compliance [2]. The candidate drug that is able to be formulated into sustained release dosage form must possess some of the criteria, including having a short half-life, high-frequent usage of drugs, and low dose drugs, which are preferred [3]. There are several methods that can be utilised to sustain the drug release, including the incorporation of drugs into hydrophobic materials, coating with swelling polymers, and encapsulating the drug molecule with certain polymers [4].
Liquisolid technology is a novel and promising method to produce a freeflowing and compressible dry powder using simple physical mixing of carrier and coating materials incorporated into a non-volatile liquid containing drugs [5]. The type of liquid used in this system is mainly nonvolatile liquid, water-miscible with high boiling temperatures, such as propylene glycol, polyethylene glycols, glycerine, or polysorbate [5]. The drug powder is incorporated in the liquid resulting in liquid medication, either a drug solution or a drug suspension. After the carrier powder is saturated with the liquid medication, a liquid layer formed on the particle surface is adsorbed by the coating material. Consequently, a dry, free-flowing, and compressible powder is obtained [6]. This technology is initially used in the purpose of improving drug solubility of poorly water-soluble drugs by using water-soluble polymer [7-9]. However, this method has been developed and the same principle was utilised to prepare sustained release formulation using hydrophobic polymers [5, 10]. This method is preferred due to its simplicity, versatility, and low cost [2].

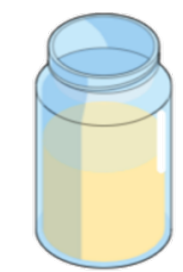

Liquid medication

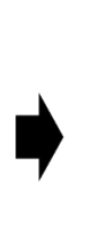

Incorporation of liquid to carrier particles

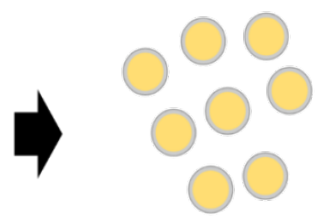

Liquid layer on particle surface (saturated with liquid)

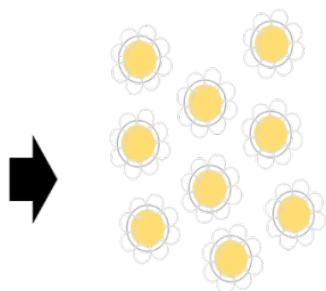

Adsorption of the liquid layer by coating material resulting in dry and free flow powder

Fig. 1: Schematic representation of liquisolid system

Propranolol, in the form of hydrochloride salt, is a suitable drug for sustained release formulation. It is one of the $\beta$-blocker antihypertension drugs with a dose of $40 \mathrm{mg}$ twice daily [11].
Propranolol is classified in the Biopharmaceutical Classification System (BCS) Class I with high solubility and high permeability which lead to fast elimination out the body. Additionally, it has low 
oral bioavailability that is only $25 \%$ reached the systemic circulation after a single administration. This results in the short half-life of propranolol which is 3 to $6 \mathrm{~h}[2,12]$. Consequently, the frequent dose is needed to maintain the therapeutic dose and efficacy of treatment. Nowadays, propranolol is available as immediate and controlled release oral dosage forms. Previously, Javadzadeh, et al. (2008) has reported the successful approach of propranolol hydrochloride formulation using liquisolid technique for sustained release delivery. However, in the previous studies, only one type of non-volatile liquid was used in each formulation [2]. Therefore, in the present work, the combination of two types of non-volatile liquid, namely propylene glycol and polysorbate 80 , in certain ratios were used in the liquisolid formulations, which were further evaluated on the physicochemical properties and dissolution profile.

\section{MATERIALS AND METHODS}

\section{Materials}

Propranolol HCl (PPH) was obtained from PT. Kimia Farma (Jakarta, Indonesia), Eudragit ${ }^{\circledR}$ RL, Aerosil ${ }^{\circledR}$, and hydroxypropyl methylcellulose (HPMC) were purchased from CV. Asian (Jakarta, Indonesia), polysorbate 80 was obtained from CV. Sentana (Makassar, Indonesia). All other materials were of analytical grade.

\section{Application of mathematical model for the design of liquisolid} granules

A mathematical model was introduced by Spireas et al. to design the formulation of liquisolid system $[6,13]$. In the present work, propylene glycol and polysorbate 80 were used as liquid vehicles, Eudragit ${ }^{\circledR}$ RL and Aerosil ${ }^{\circledR}$ were used as the carrier and coating materials, respectively. Additionally, HPMC was used as a retardant agent. The ratio of the carrier and coating materials, also known as the excipients ratio $(R)$, was 20 . It has been acknowledged that a powder can only retain a certain amount of liquid, termed as liquid load factor, while still meet the acceptance flow and compression properties.

The excipients ratio $(R)$ of powder is defined in the following equation:

$$
\mathrm{R}=\frac{\mathrm{Q}}{\mathrm{q}} \ldots . . . . \text { Equation }(1)
$$

Where $Q$ is the amount of the carrier material and $q$ is the amount of the coating material.

Liquid load factor $\left(L_{f}\right)$ is the ratio between the weight of liquid medication $(W)$ and the carrier material $(Q)$ presents in the system, as written in the equation below:

$$
\mathrm{L}_{\mathrm{f}}=\frac{\mathrm{W}}{\mathrm{Q}} \ldots \ldots . . . \text { Equation }(2)
$$

\section{Formulation of liquisolid granules of propranolol $\mathrm{HCl}$}

The liquisolid granules were prepared by firstly dispersing PPH in the liquid carrier, as illustrated in fig. 2. The carrier and coating materials, Eudragit $^{\circledR}$ RL and Aerosi $\AA^{\circledR}$, respectively, were subsequently incorporated into drug suspension resulting in a homogenous powder mixture. Furthermore, HPMC solution as the retardant agent was added to form a plastic mass. Finally, the mass was sieved using mesh no. 14 in order to obtain fine granules. The drying process of granules was performed at $50{ }^{\circ} \mathrm{C}$ for $3 \mathrm{~d}$ before being evaluated. The formulations of liquisolid granules of PPH were presented in table 1.

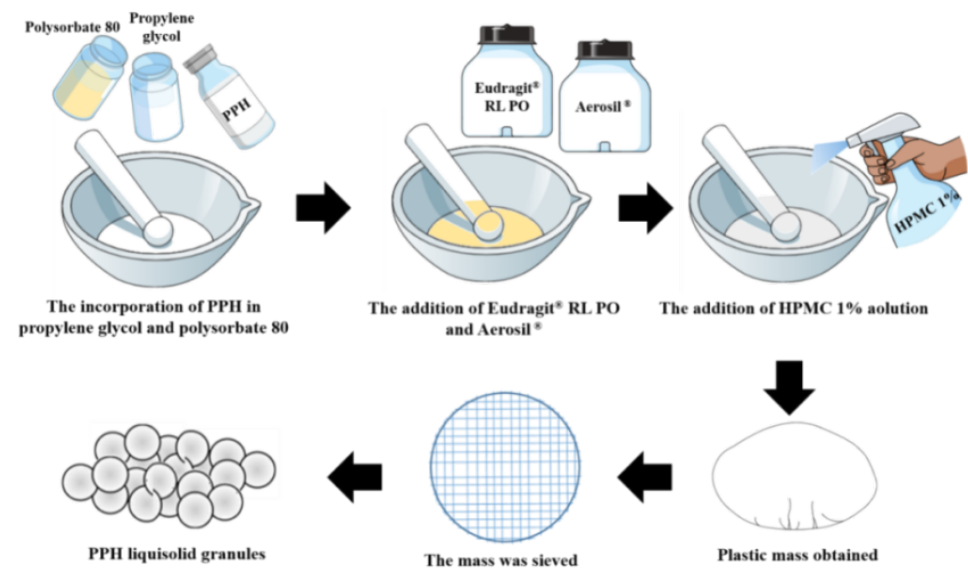

Fig. 2: Illustration of the steps in the formulation of PPH liquisolid

\begin{tabular}{|c|c|c|c|c|c|}
\hline & F1 & F2 & F3 & F4 & F5 \\
\hline PPH (g) & 1.2 & 1.2 & 1.2 & 1.2 & 1.2 \\
\hline Eudragit ${ }^{\circledR}$ RL (g) & 6.0 & 6.0 & 6.0 & 6.0 & 6.0 \\
\hline $\operatorname{Aerosil}^{\circledR}$ (g) & 0.3 & 0.3 & 0.3 & 0.3 & 0.3 \\
\hline Propylene glycol (g) & 3.0 & 2.0 & 1.5 & 1.0 & - \\
\hline Polysorbate 80 (g) & - & 1.0 & 1.5 & 2.0 & 3.0 \\
\hline HPMC 1\% (ml) & 4.0 & 4.0 & 4.0 & 4.0 & 4.0 \\
\hline
\end{tabular}

Table 1: Formulation of PPH liquisolid granules

All formulations contained liquid load factor $=0.7$ and excipient ratio $=20$

\section{Physicochemical evaluation}

\section{Loss on drying (LOD) evaluation}

LOD was used to determine the percentage of water contained in the granules by weighing the granules before and after the drying process. LOD was calculated using Equation (3):

$$
\text { LOD }(\%)=\frac{\text { wet granules weight-dry granules weight }}{\text { wet granules weight }} \ldots . . . \text { Equation (3) }
$$

\section{Flow rate and angle of repose evaluation}

As liquisolid technology is used to prepare a free-flowing powder, the flow rate and angle of repose were considerably important to be evaluated to determine the flowing properties of the granules. A flow rate evaluation was carried out by counting the flowing time of $5 \mathrm{~g}$ of granules from the determined height. Moreover, the angle of repose evaluation by measuring the angle of the formed pile of granules. Both evaluations were calculated using Equation (4) and (5) [14]: 


$$
\begin{gathered}
\text { Flow rate } v=\frac{m}{t} \ldots . . \text { Equation (4) } \\
\text { Angle of repose } \theta=\tan ^{-1}\left(\frac{2 \mathrm{~h}}{\mathrm{~d}}\right) \ldots \ldots . \text { Equation (5) }
\end{gathered}
$$

Where $\mathrm{v}$ is the flow rate, $\mathrm{m}$ is the mass of granules, $\mathrm{t}$ is the flowing time of granules, 0 is the angle of repose, $h$ is the height of the pile, and $d$ is the length of the pile base.

\section{Fourier transform infrared (FT-IR) spectroscopy}

FT-IR spectroscopy was conducted in order to detect any interactions between drugs and excipients. The IR spectra of the samples were recorded between $4500-250 \mathrm{~cm}^{-1}$ using 32 scans with a resolution of $4.0 \mathrm{~cm}^{-1}$ at room temperature $\left(20^{\circ} \mathrm{C}\right)$.

\section{Drug content analysis}

An amount of $10 \mathrm{mg}$ of granules was dissolved in simulated gastric fluid up to $10 \mathrm{ml}$ before being sonicated for $10 \mathrm{~min}$. The solution was subsequently filtered, and $1.25 \mathrm{ml}$ of the filtrate was diluted with simulated gastric fluid up to $5 \mathrm{ml}$. The final solution was measured using a spectrophotometer UV-Visible at $290 \mathrm{~nm}$.

\section{Dissolution studies}

An amount of granules, equivalent to $40 \mathrm{mg} \mathrm{PPH}$, was tested using dissolution apparatus type 1 (rotating basket). The dissolution test was performed in two different dissolution media, $900 \mathrm{ml}$ of simulated gastric fluid and $900 \mathrm{ml}$ of simulated intestinal fluid. All the experiments were performed at $50 \mathrm{rpm}$ at $37^{\circ} \mathrm{C}$. An aliquot of $5 \mathrm{ml}$ was taken using millipore after 30 and $60 \mathrm{~min}$ in a simulated gastric fluid medium and after 1, 2, 3, 4, 5, 6 and $7 \mathrm{~h}$ in simulated intestinal fluid. Each sample was diluted using the suitable dissolution medium up to $10 \mathrm{ml}$. The sample taken was replaced with fresh dissolution medium. The amount of PPH in the sample was measured using a spectrophotometer UV-Visible at $290 \mathrm{~nm}$. All experiments were conducted in triplicates.

\section{Drug release kinetics determination}

In order to describe the dissolution profiles of liquisolid granules, the data obtained were analysed using the mathematical equation of each kinetic model [15-18]:

$$
\begin{gathered}
\text { Zero order: } C_{t}=C_{o}+k_{0} t \ldots \ldots . \text { Equation (6) } \\
\text { First order: } \ln C_{t}=\ln C_{o}+k_{1} t \quad \ldots . . \text { Equation (7) } \\
\text { Higuchi model: } C_{t}=k_{H} \sqrt{t} \ldots \ldots . . \text { Equation (8) } \\
\text { Hixson-Crowell model: } C_{t}^{1 / 3}=C_{0}^{1 / 3}+k_{H C} t \quad \text { Equation (9) }
\end{gathered}
$$

Where $C_{t}$ is the concentration of drug dissolved in time $t, C_{o}$ is the initial concentration of the drug, $k_{0}$ is the zero order release constant, $\mathrm{k}_{1}$ is the first order release constant, $\mathrm{k}_{\mathrm{H}}$ is the Higuchi model constant, and $\mathrm{k}_{\mathrm{HC}}$ is the Hixson-Crowell model constant. The value of correlation coefficient $\left(\mathrm{r}^{2}\right)$ was used to determine the bestfitted release model of each formulation.

\section{Data analysis}

The difference $\left(f_{1}\right)$ and similarity $\left(f_{2}\right)$ factors were calculated in order to compare the release profiles of each liquisolid formulation. The difference factor $\left(f_{1}\right)$ was used to measure the percentage of difference and percent error between two dissolution profiles over all time points. Besides, the similarity factor $\left(f_{2}\right)$ is a logarithmic square-root transformation of differences between two formulations referred as the test and the reference. Equation (10) and (11) were used to calculate $f_{1}$ and $f_{2}$, respectively [19].

$$
\begin{gathered}
\mathrm{f}_{1}=\left\{\left[\sum_{\mathrm{t}=1}^{\mathrm{n}}\left(\mathrm{R}_{\mathrm{t}}-\mathrm{T}_{\mathrm{t}}\right)\right] /\left[\sum_{\mathrm{t}=1}^{\mathrm{n}} \mathrm{R}_{\mathrm{t}}\right]\right\} \mathrm{x} 100 \\
\mathrm{f}_{2}=50 \mathrm{x} \log \left\{\left[1+(1 / \mathrm{n}) \sum_{\mathrm{t}=1}^{\mathrm{n}}\left(\mathrm{R}_{\mathrm{t}}-\mathrm{T}_{\mathrm{t}}\right)^{2}\right]^{-0.5} \mathrm{x} 100\right\}
\end{gathered}
$$

Where $\mathrm{n}$ is the number of sampling time, $R_{t}$ is the reference dissolution percentage at time $t$, and $T_{t}$ is the test dissolution percentage at time $t$. Two dissolution profiles are considered similar if the value of $f_{1}$ is lower than 15 (in the range of 0-15) and the value of $f_{2}$ is more than 50 (in the range of 50-100).

\section{RESULTS AND DISCUSSION}

\section{Formulation of liquisolid granules of propranolol $\mathrm{HCl}$}

Liquisolid method was chosen as the most suitable method to prepare sustained-release granules for oral delivery due to its simplicity in process and availability of the materials [20]. In this research, PPH was selected as the drug model because of its good solubility and good permeability. This caused PPH to become the most suitable candidate for prolonged-release delivery. Besides, its massive use as an antihypertension drug was another consideration for better improvement in efficacy and patient compliance [21].

Briefly, the process involved in the preparation of liquisolid granules was similar to wet granulation method in tablet preparation due to the incorporation of liquid, in this case was HPMC $1 \%$ solution, resulting in forming a plastic mass which was further sieved into granules. Eudragit ${ }^{\circledR}$ RL is one of the polymethacrylates that is commonly used as a water-insoluble film coating for sustained release delivery [22]. In the liquisolid system, the physicochemical properties of Eudragit ${ }^{\circledR}$ RL met the requirement as carrier material, to absorb the drug liquid and modify the properties of PPH from water-soluble to water-insoluble. Coating materials with high adsorptive properties and large surface area such as Aerosil ${ }^{\circledR}$ were able to produce free-flowing dry liquisolid powder. The addition of HPMC increased the retardation effect of this system [23].

\section{Physicochemical evaluation}

\section{Loss on drying (LOD) evaluation}

LOD percentage was calculated to determine the amount of water loss in the drying process. This parameter indicated the varied concentration of propranolol in the system, which was subsequently calculated in the drug content evaluation. The amount of water loss in the drying process was more than presented in the designed formulations. This might be caused by the hygroscopicity of both propylene glycol and polysorbate 80 [22]. Hence, the drying process was considerably important to ensure the granules was dry prior to evaluation.

Table 2: The results of LOD, flow rate, and angle of repose evaluations

\begin{tabular}{llll}
\hline & \%LOD & Flow rate $(\mathrm{g} / \mathbf{s})$ & Angle of repose $\left({ }^{\circ}\right)$ \\
\hline F1 & 46.88 & 1.09 & 24.52 \\
F2 & 42.14 & 1.51 & 28.85 \\
F3 & 38.73 & 1.10 & 30.20 \\
F4 & 35.71 & 2.07 & 26.52 \\
F5 & 26.15 & 4.19 & 20.96 \\
\hline
\end{tabular}

\section{Flow rate and angle of repose evaluation}

The flow properties of powder or granules were essential to be determined because it was going to be compressed into tablets or filled in capsules. This evaluation was performed by measuring the flow rate. The method used in this study was simple and easy, yet adequate by measuring the time taken of an amount of granules to flow from a tunnel at a certain height to a flat surface. The higher flow rate showed better flow properties. Furthermore, angle of repose can be another parameter to evaluate the granules flow properties. Granules with the angle of repose of less than $30{ }^{\circ} \mathrm{C}$ indicated very good flow properties [24]. From table 2, it can be seen that all formulations met the requirements, which means the granules prepared are free-flowing. 

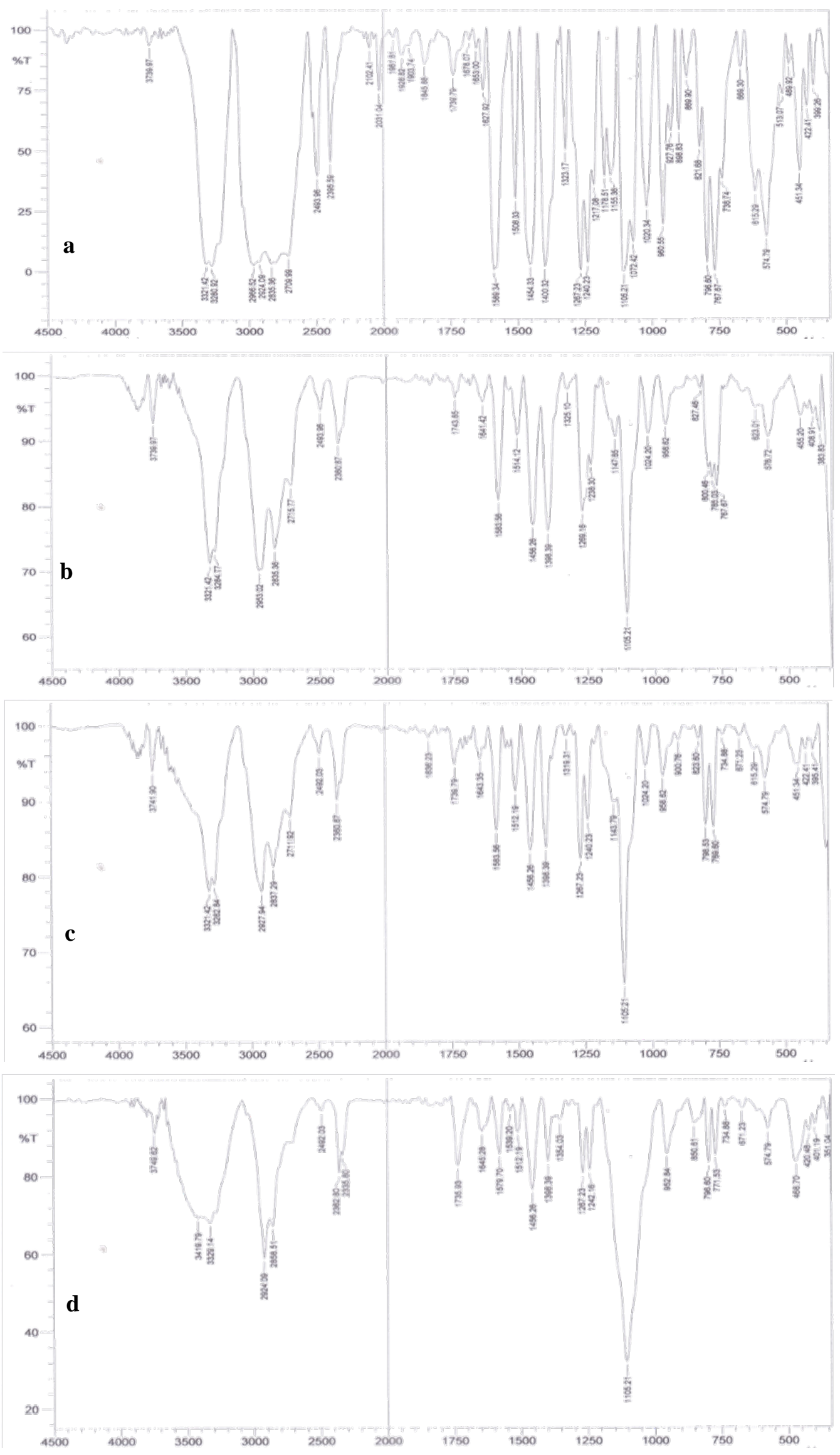


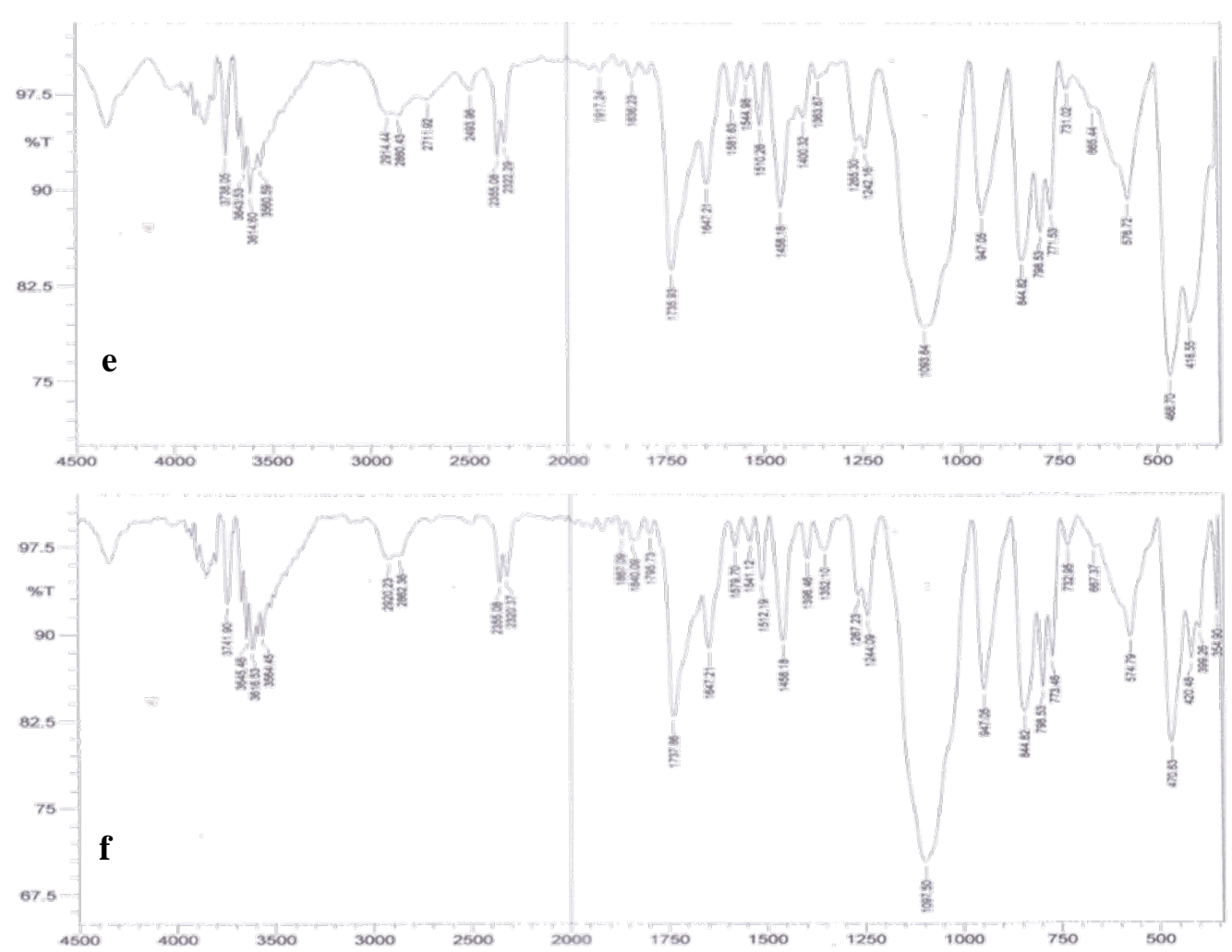

Fig. 3: FTIR spectra of (a) propranolol hydrochloride, (b) F1, (c) F2, (d) F3, (e) F4, and (f) F5, respectively

\section{FT-IR spectroscopy}

Interaction between drug and excipients was observed using FT-IR spectroscopy. The specific peaks detected represented specific chemical bonds in the drug or mixture. Based on its chemical structure, PPH has several typical peaks as can be seen in fig. 3. Two peaks appeared at 3321.42 and $3280.92 \mathrm{~cm}^{-1}$ represented secondary amine and hydroxyl groups, respectively. Additionally, the chemical bond of $\mathrm{C}-\mathrm{H}$ as methyl asymmetric group appeared at $2966.52 \mathrm{~cm}^{-1}$ and the absorption of aromatic ring band was at $1589.34 \mathrm{~cm}^{-1}$. The other chemical groups, including aryl alkyl ether and $\mathrm{C}-\mathrm{H}$ bond in the aromatic ring was observed at 1267.23 and $796.6 \mathrm{~cm}^{-1}$, respectively $[25,26]$. All these peaks were also observed in the spectra of all liquisolid formulations without major shifting. Therefore, it was confirmed that no chemical interaction was found between PPH and other excipients. This means that there is no change in the chemical structure of PPH, which indicated efficacy in the therapeutic effect of PPH.

\section{Drug content analysis}

In the liquisolid system, the drug is entrapped and surrounded by the hydrophobic polymer. Therefore, the sonication process was important to ensure all the drug in the granules was dissolved prior to measurement. The drug content analysis was carried out in order to fig. out the amount of PPH in the liquisolid system. It can be seen that the percentage of PPH in the granules was in the range between $7-9 \%$. The varied concentration obtained might be caused by the different percentage on loss on drying process. However, as the results of this evaluation were used as the basis to calculate the drug released in the in vitro release studies, the varied results were not highly concerned.

Table 3: Percentage of the drug content of $\mathrm{PPH}$ liquisolid formulations (mean $\pm S D, n=3$ )

\begin{tabular}{ll}
\hline Formula & \%Drug content $(\mathbf{w} / \mathbf{w})$ \\
\hline F1 & $9.33 \pm 0.02$ \\
F2 & $8.80 \pm 0.32$ \\
F3 & $7.89 \pm 0.27$ \\
F4 & $7.93 \pm 0.44$ \\
F5 & $7.09 \pm 0.37$ \\
\hline
\end{tabular}

\section{Dissolution studies}

In this study, the dissolution studies were conducted in two different dissolution media, namely simulated gastric fluid and simulated intestinal fluid. It is important to note that the liquisolid system prepared in this research was not designed for mucoadhesive or floating system. Accordingly, the use of two dissolution media aimed to represent the gastric and intestinal, where the drug was expected to release.

Overall, the results show that all formulations were able to sustain until a total of $8 \mathrm{~h}$. This indicated that the presence of Eudragit ${ }^{\circledR} \mathrm{RL}$ as a hydrophobic polymer was able to completely to coat the drug molecule resulting in prolonged drug release [8]. Moreover, the swelling properties of HPMC was able to form a gel barrier that controlled the release of PPH $[27,28]$. Specifically, all the liquisolid formulations released less than $50 \%$ after 1 hour in the simulated gastric fluid, with the lowest released percentage was given by F4. In contrast, F5 provided the highest amount of PPH released, $47.13 \pm 0.35 \%$ after $60 \mathrm{~min}$ in the first media. Furthermore, the trend of results obtained in the simulated intestinal fluid was slightly similar to those in the simulated gastric fluid.

After 1 hour, there was almost no major difference in the percentage of drug released of all formulation. However, it can be clearly seen in fig. 3, F4 was able to provide a more sustained release of PPH compared to other formulations at the seventh hour. Interestingly, F5 showed the same percentage as F4 at the last time point. This indicated that the combination of liquid carrier and the $\mathrm{pH}$ of the dissolution media played an important role in the dissolution studies. It was reported that the presence of liquid vehicle acting as a plasticizer was able to decrease the glass transition temperature of Eudragit ${ }^{\circledR} \mathrm{RL}$, resulting in retardation effect of liquisolid granules $[5,22]$. By varying the ratio of liquid vehicle, it was obtained that the liquisolid system containing propylene glycol and polysorbate 80 with the ratio of $1: 2$ provided the least dissolution release rate. As reported in the previous studies, the solubility of the drug in the liquid carrier also affected the release of drug [29]. The solubility of PPH in propylene glycol was higher than in polysorbate 80 , which lead to the more undissolved drug in the liquid carrier [2]. This subsequently increased the retardation effect in the liquisolid granules. 
Based on the difference and similarity factors calculation, as shown in table 4 , the dissolution profiles of all formulations were found to be similar one another since all the values of $f_{1}$ were less than 15 and the values of $f_{2}$ were higher than 50 . Therefore, this suggested that there was no significant difference in the release profile of the liquisolid system, however, this also showed the granules prepared were able to sustain the PPH release for $8 \mathrm{~h}$ with the lowest rate provided by F4.

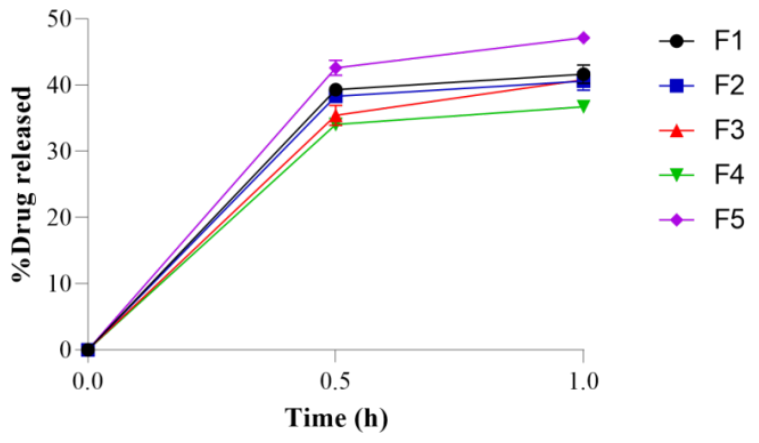

a

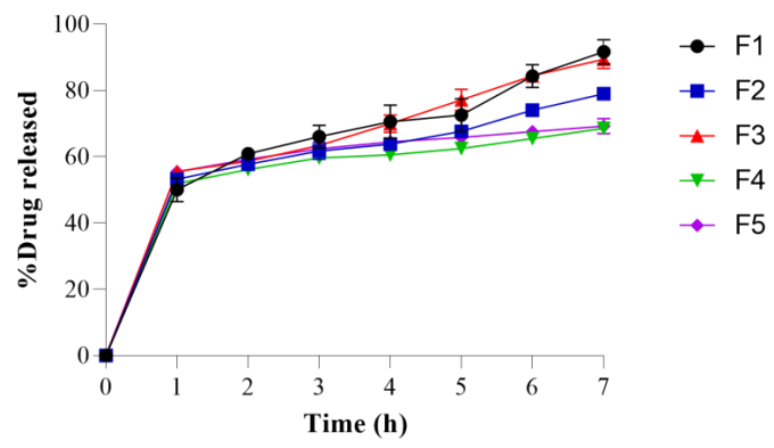

b

Fig. 4: Dissolution profiles of PPH liquisolid granules in the (a) simulated gastric fluid and (b) simulated intestinal fluid $($ mean $\pm S D, n=3$ )
Table 4: Difference $\left(f_{1}\right)$ and similarity $\left(f_{2}\right)$ factor of each dissolution profile of liquisolid formulations

\begin{tabular}{llll}
\hline Curve 1 & Curve 2 & F1 & F2 \\
\hline F1 & F2 & 4.56 & 68.91 \\
F1 & F3 & 2.38 & 82.04 \\
F1 & F4 & 9.78 & 54.00 \\
F1 & F5 & 5.76 & 62.74 \\
F2 & F3 & 4.33 & 70.28 \\
F2 & F4 & 5.80 & 67.23 \\
F2 & F5 & 4.75 & 70.84 \\
F3 & F4 & 8.93 & 55.43 \\
F3 & F5 & 6.55 & 62.24 \\
F4 & F5 & 9.43 & 58.73 \\
\hline
\end{tabular}

\section{Drug release kinetics determination}

Based on the dissolution released data, the drug release kinetics of each formulation was able to be determined. Each of the dissolution plots was fitted to each of release model equation, including zero order, first order, Higuchi model, and Hixson-Crowell model. The kinetics profile was subsequently able to interpret the release mechanism of the formulation. The degree of correlation coefficient $\left(\mathrm{r}^{2}\right)$, which was closest to 1 , was chosen as the best-fitted to the mathematical equation model, as shown in table 3 . The results show that F1 and F3 were best fitted to first order kinetics. This suggested that the drug was transported through non-Fickian diffusion, where the release rate depends on the concentration of the drug [30]. This model was also followed by the formulation of a water-soluble drug in porous matrices [31]. Besides, F1, F4, and F5 were best fitted to the Higuchi model. According to Higuchi square root model, the drug release of these formulations followed the diffusion controlled release mechanism [32].

The results of this study have shown the feasibility of the preparation of PPH liquisolid granules for better sustained release profiles. Besides, it has been suggested that the combination of liquid vehicles could be utilised to obtain the optimum formulation with the desired dissolution profiles. Therefore, following these promising findings, further studies such as the formulation of pharmaceutical dosage forms using the obtained granules are required to object the clinical usage. Furthermore, toxicity and in vivo studies using suitable animal models should also be performed to generate a complete review of the potential of this proposed system.

Table 5: The value of correlation coefficient of each dissolution profile of liquisolid formulation

\begin{tabular}{|c|c|c|c|c|}
\hline \multirow[t]{2}{*}{ Formula } & \multicolumn{4}{|c|}{ Correlation coefficient value $\left(r^{2}\right)$} \\
\hline & Zero order & First order & Higuchi model & Hixson-crowell model \\
\hline F1 & 0.7411 & 0.9395 & 0.9234 & 0.9053 \\
\hline $\mathrm{F} 2$ & 0.6928 & 0.8717 & 0.8937 & 0.8144 \\
\hline F3 & 0.7414 & 0.9501 & 0.9219 & 0.8990 \\
\hline F4 & 0.6560 & 0.7803 & 0.8652 & 0.7399 \\
\hline F5 & 0.5945 & 0.7405 & 0.8282 & 0.6944 \\
\hline
\end{tabular}

\section{CONCLUSION}

Liquisolid technology is one of the simple and promising methods which was successfully adapted to prepare sustained release dosage forms. In this study, propranolol hydrochloride (PPH) was selected as the drug of choice due to its high-water solubility, low dose, short half-life, and frequent dosing. The combination of propylene glycol and polysorbate 80 were used as the liquid vehicles. The prepared formulations possessed first-order and Higuchi model kinetics. There was no significant difference observed in the dissolution profile, as all the formulations were able to sustain the drug release over $8 \mathrm{~h}$. Therefore, the presented technique can be a promising solution for the prolonged release formulation of water-soluble drugs. In addition to that, further comprehensive studies are considerably required, including the formulation of solid dosage forms, toxicity and in vivo studies using suitable animal models.

\section{FUNDING}

Nil

\section{AUTHORS CONTRIBUTIONS}

All the authors have contributed equally.

\section{CONFLICT OF INTERESTS}

The authors have no conflict of interest to declare.

\section{REFERENCES}

1. Bose S, Kaur A, Sharma SK. A review on advances of sustained release drug delivery system. Int Res J Pharm 2013;4:1-5.

2. Javadzadeh Y, Musaalrezaei L, Nokhodchi A. Liquisolid technique as a new approach to sustain propranolol 
hydrochloride release from tablet matrices. Int J Pharm 2008;362:102-8.

3. Gupta M, Brijesh R. A review on: sustained release technology. Int J Ther Appl 2012;8:18-23.

4. Badadhe S, Gawali V, Bhalsing M, Jadhav B. Sustained release dosage forms: an overview. Int J Innov Pharm Sci Res 2016;4:1194-211.

5. NP K, MT S, DH S, PK S. A review on liquisolid systems. J Drug Delivery Ther 2014;4:25-31.

6. Pawar JD, Jagtap RS, Doijad RC, Pol SV, Desai JR, Jadhav VV, et al. Liquisolid Compacts: a promising approach for solubility enhancement. J Drug Delivery Ther 2017:7:6-11.

7. Mokashi AA, Gaikwad SL. Formulation and evaluation of liquisolid compacts of lornoxicam. Int J Pharm Pharm Sci 2019;11:33-7.

8. Anil A, Thomas L, Sudheer P. Liquisolid compacts: an innovative approach for dissolution enhancement. Int J Pharm Pharm Sci 2018;10:11-7.

9. Jassimp ZE. Formulation and evaluation of furosemide liquisolid compact. Int J Appl Pharm 2017;9:39-48.

10. Nokhodchi A, Hentzschel CM, Leopold CS. Drug release from liquisolid systems: speed it up, slow it down. Expert Opin Drug Delivery 2011;8:191-205.

11. Sweetman SC. editor. Martindale: The Complete Drug Reference. $36^{\text {th }}$ ed. London: Pharmaceutical Press; 2009.

12. Yadav A, Jain DK. Formulation development and characterization of propranolol hydrochloride microballoons for gastroretentive floating drug delivery. Afr J Pharm Pharmacol 2011;5:1801-10.

13. Panda S, Varaprasad R, Priyanka K, Swain RP. Liquisolid technique: a novel approach for dosage form design. Int J Appl Pharm 2017;9:8-14.

14. Shah RB, Tawakkul MA, Khan MA. Comparative evaluation of flow for pharmaceutical powders and granules. AAPS PharmSciTech 2008;9:250-8.

15. Holowka EP, Bhatia SK. Controlled release systems. In: Drug Delivery: Materials Design and Clinical Perspective; 2014. p. 8-62.

16. Permana AD, Paredes AJ, Volpe Zanutto F, Anjani QK, Utomo E, Donnelly RF. Dissolving microneedle-mediated dermal delivery of itraconazole nanocrystals for improved treatment of cutaneous candidiasis. Eur J Pharm Biopharm 2020;154:50-61.

17. Permana AD, Tekko IA, McCrudden MTC, Anjani QK, Ramadon D, McCarthy HO, et al. Solid lipid nanoparticle-based dissolving microneedles: A promising intradermal lymph targeting drug delivery system with potential for enhanced treatment of lymphatic filariasis. J Controlled Release 2019;316:34-52.

18. Permana AD, McCrudden MTC, Donnelly RF. Enhanced intradermal delivery of nanosuspensions of antifilariasis drugs using dissolving microneedles: a proof of concept study. Pharmaceutics 2019;11:1-22.

19. Costa P, Lobo JMS. Modeling and comparison of dissolution profiles. Eur J Pharm Sci 2001;13:123-33.

20. Lu M, Xing H, Jiang J, Chen X, Yang T, Wang D, et al. Liquisolid technique and its applications in pharmaceutics. Asian J Pharm Sci 2017;12:115-23.

21. Sahoo J, Murthy PN, Biswal S, Sahoo SK, Mahapatra AK. Comparative study of propranolol hydrochloride release from matrix tablets with Kollidon ${ }^{\circledR S R}$ or hydroxy propyl methyl cellulose. AAPS PharmSciTech 2008;9:577-82.

22. Rowe RC, Sheskey PJ, Quinn ME. editors. Handbook of Pharmaceutical Excipients. Sixth. London: Pharmaceutical Press; 2009.

23. Balaji A, Umashankar MS, Kavitha B. Liquisolid technology-a latest review. Int J Appl Pharm 2014;6:11-9.

24. The United States Pharmacopeial Convention. USP 35. Vol. 35 U. S. Pharmacopoeia; 2012. p. 801-4.

25. Chaturvedi K, Umadevi S, Vaghani S. Floating matrix dosage form for propranolol hydrochloride based on gas formation technique: development and in vitro evaluation. Sci Pharm 2010;78:927-39.

26. Hernawan, Nurhayati $S$, Nisa K, Indrianingsih AW, Darsih C, Kismurtono M. Formulation and in vitro study of propranolol hydrochloride controlled release from carboxymethyl chitosanbased matrix tablets. Indones J Chem 2013;13:242-7.

27. Shoaib MH, Tazeen J, Merchant HA, Yousuf RI. Evaluation of drug release kinetics from ibuprofen matrix tablets using HPMC. Pak J Pharm Sci 2006;19:119-24.

28. Siepmann J, Peppas NA. Modeling of drug release from delivery systems based on hydroxypropyl methylcellulose (HPMC). Adv Drug Delivery Rev 2012;64:163-74.

29. Adibkia K, Shokri J, Barzegar Jalali M, Solduzian M, Javadzadeh Y. Effect of solvent type on retardation properties of diltiazem $\mathrm{HCl}$ form liquisolid tablets. Colloids Surfaces B Biointerfaces 2014;113:10-4.

30. Wojcik Pastuszka D, Krzak J, Macikowski B, Berkowski R, Osiński B, Musiał W. Evaluation of the release kinetics of a pharmacologically active substance from model intra-articular implants replacing the cruciate ligaments of the knee. Materials (Basel) 2019;12:1208.

31. Paarakh MP, Jose PANI, Setty CM, Peter GV. Release kineticsconcepts and applications. Int J Pharm Res Technol 2019;8:1220.

32. Gouda R, Baishya H, Qing Z. Application of mathematical models in drug release kinetics of carbidopa and levodopa ER tablets. J Dev Drugs 2017;6:1-8. 